\title{
An assessment of the measurement of the Lense-Thirring effect in the Earth gravity field, in reply to: "On the measurement of the Lense-Thirring effect using the nodes of the LAGEOS satellites, in reply to "On the reliability of the so far performed tests for measuring the Lense-Thirring effect with the LAGEOS satellites" by L. Iorio," by I. Ciufolini and E. Pavlis
}

\author{
L. Iorio \\ Viale Unità di Italia 68, 70125 \\ Bari, Italy \\ tel./fax 00390805443144 \\ e-mail: lorenzo.iorio@libero.it
}

\begin{abstract}
In this paper we reply to recent claims by Ciufolini and Pavlis about certain aspects of the measurement of the general relativistic Lense-Thirring effect in the gravitational field of the Earth. I) The proposal by such authors of using the existing satellites endowed with some active mechanisms of compensation of the non-gravitational perturbations as an alternative strategy to improve the currently ongoing Lense-Thirring tests is unfeasible because of the impact of the uncancelled even zonal harmonics of the geopotential and of some time-dependent tidal perturbations. II) It is shown that their criticisms about the possibility of using the existing altimeter Jason- 1 and laser-ranged Ajisai satellites are groundless. III) Ciufolini and Pavlis also claimed that we would have explicitly proposed to use the mean anomaly of the LAGEOS satellites in order to improve the accuracy of the Lense-Thirrring tests. We prove that it is false. In regard to the mean anomaly of the LAGEOS satellites, Ciufolini himself did use such an orbital element in some previously published tests. About the latest test performed with the LAGEOS satellites, IV) we discuss the cross-coupling between the inclination errors and the first even zonal harmonic as another possible source of systematic error affecting it with an additional $9 \%$ bias. V) Finally, we stress the weak points of the claims about the origin of the two-nodes LAGEOS-LAGEOS II combination used in that test.
\end{abstract}


Keywords: Lense-Thirring effect; Earth gravity field; active spacecraft; passive spacecraft; polar orbits; mean anomaly

\section{Introduction}

The post-Newtonian Lense-Thirring (LT) effect is one of the few predictions of the Einsteinian General Theory of Relativity (GTR) for which a direct, clean and undisputable test is not yet available with a really satisfying precision $(\leq 1 \%)$.

According to Einstein, the action of the gravitational potential $U$ of a given distribution of mass-energy on a particle moving with velocity $\boldsymbol{v}$ is described by the coefficients $g_{\mu \nu}, \mu, \nu=0,1,2,3$, of the space-time metric tensor. They are determined, in principle, by solving the fully non-linear field equations of GTR for the considered mass-energy content. These equations can be linearized in the weak-field $\left(U / c^{2}<<1\right.$, where $c$ is the speed of light in vacuum) and slow-motion $(v / c<<1)$ approximation (Mashhoon et al., 2001; Ruggiero and Tartaglia 2002), valid throughout the Solar System, and look like the equations of the linear Maxwellian electromagnetism. Among other things, a noncentral, Lorentz-like force

$$
\boldsymbol{F}_{\mathrm{LT}}=-2 m\left(\frac{\boldsymbol{v}}{c}\right) \times \boldsymbol{B}_{g}
$$

acts on a moving test particle of mass $m$. It is induced by the postNewtonian component $\boldsymbol{B}_{g}$ of the gravitational field in which the particle moves. $\boldsymbol{B}_{g}$ is related to the mass currents of the mass-energy distribution of the source and comes from the off-diagonal components $g_{0 i}, i=1,2,3$ of the metric tensor. Thanks to such an analogy, the ensemble of the gravitational effects induced by mass displacements is also named gravitomagnetism. For a central rotating body of proper angular momentum $\boldsymbol{L}$ the gravitomagnetic field is

$$
\boldsymbol{B}_{g}=\frac{G\left[3 \boldsymbol{r}(\boldsymbol{r} \cdot \boldsymbol{L})-r^{2} \boldsymbol{L}\right]}{c r^{5}} .
$$

One of the consequences of eq. (11) and eq. (2) is a gravitational spinorbit coupling. Indeed, if we consider the orbital motion of a particle in the gravitational field of a central spinning mass, it turns out that the longitude of the ascending node $\Omega$ and the argument of pericentre $\omega$ of the orbit of the

test particle are affected by tiny secular precessions $\dot{\Omega}_{\mathrm{LT}}, \dot{\omega}_{\mathrm{LT}}$ (Lense and Thirring, 1918; Barker and O'Connell, 1974; Cugusi and Proverbio, 1978; 
Soffel, 1989; Ashby and Allison, 1993; Iorio, 2001a)

$$
\dot{\Omega}_{\mathrm{LT}}=\frac{2 G L}{c^{2} a^{3}\left(1-e^{2}\right)^{\frac{3}{2}}}, \dot{\omega}_{\mathrm{LT}}=-\frac{6 G L \cos i}{c^{2} a^{3}\left(1-e^{2}\right)^{\frac{3}{2}}},
$$

where $a, e$ and $i$ are the semimajor axis, the eccentricity and the inclination, respectively, of the orbit and $G$ is the Newtonian gravitational constant. Note that in their original paper Lense and Thirring (1918) used the longitude of pericentre $\varpi \equiv \Omega+\omega$.

The gravitomagnetic force may have strong consequences in many astrophysical and astronomical scenarios involving, e.g., accreting disks around black holes (Stella et al., 2003), gravitational lensing and time delay (Sereno 2003; 2005a; 2005b). Unfortunately, in these contexts the knowledge of the various competing effects is rather poor and makes very difficult to reliably extract the true gravitomagnetic signal from the noisy background. E.g., attempts to measure the LT effect around black holes are often confounded by the complexities of the dynamics of the hot gas in their accretion disks. On the contrary, in the Solar System space environments the LT effect is weaker but the various sources of systematic errors are relatively well known and we have the possibility of using various artificial and natural orbiters both to improve our knowledge of such biases and to design suitable observables circumventing these problems, at least to a certain extent.

Up to now, all the performed and ongoing tests of gravitomagnetism were implemented in the weak-field and slow-motion scenarios of the Earth, the Sun and Mars gravitational fields.

Concerning the terrestrial environment, in April 2004 the GP-B spacecraft (Everitt et al., 2001) was launched. Its aim is the measurement of another gravitomagnetic effect, i.e. the precession of the spins (Schiff, 1960) of four superconducting gyroscopes carried onboard with an expected accuracy of $1 \%$ or better. According to Nordtvedt (2003), the multi-decade analysis of the Moon's orbit by means of the Lunar Laser Ranging (LLR) technique yields a comprehensive test of the various parts of order $\mathcal{O}\left(c^{-2}\right)$ of the post-Newtonian equation of motion. The existence of the LT signature as predicted by GTR would, then, be indirectly inferred from the high accuracy of the lunar orbital reconstruction. Also the radial motion of the LAGEOS satellite would yield another indirect confirmation of the existence of the LT effect (Nordtvedt, 1988). Recently, a test of the LT effect on the orbit of a test particle was performed by Ciufolini and Pavlis (2004). They analyzed the data of the laser-ranged LAGEOS and LAGEOS II satellites in the gravitational field of the Earth by using an observable proposed in (Ries 
et al., 2003a; 2003b; Iorio and Morea, 2004; Iorio, 2005a; 2006a). The total accuracy claimed by Ciufolini and Pavlis is $5-10 \%$ at $1-3$ sigma, respectively, but such estimate is controversial (Iorio, 2005b; 2006b; Lucchesi 2005) for various reasons mainly related to a realistic assessment of the impact of the static and time-varying parts of the Earth gravity field. The total error may be as large as about $20 \%$ at 1 sigma level (Iorio, 2005a; 2006b). Proposals for the use of at least one new satellite can be found, e.g., in (Ries et al., 1989); in (Iorio, 2005c) it is re-examined in the context of the recent advances in our knowledge of the terrestrial gravitational field.

In regard to the solar gravitomagnetic field, recent progresses in the orbit determination of the Solar System planets (Pitjeva, 2005) allowed, for the first time, to search for evidence of the LT precessions of the planetary orbits (Iorio, 2005d; 2005e). In fact, the general relativistic predictions are compatible with the determined extra-perihelion advances of the inner planets, but the errors are still large: a zero-effect hypothesis is compatible with them as well, although at a worse level. However, the continuous processing of accurate radar-ranging data and the ongoing/planned spacecraft-based missions to Mercury, Venus and Mars like, e.g., BepiColombo, Messenger and Venus Express should improve the robustness and the precision of such preliminary tests.

Very recently, a 6\% LT test on the orbit of the Mars Global Surveyor (MGS) spacecraft in the gravitational field of Mars has been reported (Iorio 2006c); indeed, the predictions of general relativity are able to accommodate, on average, about $94 \%$ of the measured residuals in the out-of-plane part of the MGS orbit over 5 years.

At present, there are no observational evidences pointing against the existence of the gravitomagnetic force as predicted by general relativity. Thus, the main interest in an evaluation of the accuracy of our knowledge of the LT effect as much accurate and reliable as possible mainly resides in estimating the quality of orbitography and of the gravitational and nongravitational force modelling without neglecting gravitomagnetism.

The focus of this paper is on some issues related to the LT test recently performed in the terrestrial gravitational field with the nodes of LAGEOS and LAGEOS II and on possible alternative strategies to enhance its reliability and accuracy by using some of the existing active or passive satellites. The subsequent debate has raised controversies often pursued by Ciufolini and Pavlis (2005) in a way completely inappropriate for scientific works, both for content and language.

The main points are 
- In (Ciufolini and Pavlis, 2005) it is written: "[...] one wonders why the author of Iorio $^{1}$ (2005) has not proposed the use of the GRACE satellites which not only have by far better shape and orbital stability compared to JASON but they also carry ultra precise accelerometers that measure all non-conservative accelerations, so that they are in practice "free-falling" particles in vacuum, at least to the extent that is covered by the accuracy of these instruments."

The answer is that the existing spacecraft with active mechanisms of compensation of the non-gravitational forces (CHAMP, GRACE, GPB) fly at too low altitudes in polar orbits, so that the use of their nodes, combined or not with those of the LAGEOS satellites, would greatly enhance the systematic errors both due to the uncancelled even zonal harmonic coefficients $J_{\ell}$ of the multipolar expansion of the terrestrial gravitational potential and to certain time-dependent tidal perturbations. This topic is discussed in detail in Section 2.

- In Section 3 we deal with the proposal of using also the data from the existing altimeter Jason-1 and laser-ranged Ajisai satellites (Iorio and Doornbos, 2005; Vespe and Rutigliano, 2005) to improve and enforce the accuracy and the reliability of the LT tests. Such a suggestion was only superficially criticized by Ciufolini and Pavlis (2005), without even quoting the relevant works.

- The title of Section 5 of (Ciufolini and Pavlis, 2005) is: "On the use of the mean anomaly and on the use of Jason to measure the LenseThirring effect proposed in Iorio (2004) ". Moreover, in (Ciufolini and Pavlis, 2005) it is also written that "[...] one of the most profound mistakes and misunderstandings of $\operatorname{Iorio}^{2}$ (2005) is the proposed use of the mean anomaly of a satellite to measure the Lense-Thirring effect (in some previous paper by the same author the use of the mean anomaly was also explicitly proposed)." Such claims are not correct, as it will be shown in Section 4

- In Section [5] we discuss the bias induced on the test performed with the LAGEOS satellites by the cross-coupling between the first even zonal harmonic of the geopotential and the errors in the inclinations of LAGEOS and LAGEOS II. It yields a further $9 \%$ systematic bias.

\footnotetext{
${ }^{1}$ It is the reference (Iorio, 2005b) of this paper.

${ }^{2}$ It is the reference (Iorio, 2005b) of this paper.
} 
- Finally, in Section 6 we discuss the plagiarism by Ciufolini and Pavlis (2004) about the combination of the nodes of LAGEOS and LAGEOS II (Ries et al., 2003a; 2003b; Iorio and Morea, 2004; Iorio, 2005a; 2006a) used by them and show that their claims about its origin are not exact.

\section{On the possible use of other existing active and passive spacecraft}

\subsection{The linear combination approach: a brief review}

A general description of the approach involving the reduction of the systematic error due to the mismodelling in the classical part of the terrestrial gravitational field by using suitable linear combinations of the satellites' nodes can be found, e.g., in (Iorio, 2002a; 2004; 2005b) and references therein; here we briefly recall it (see also Section 5).

Among the six Keplerian orbital elements in terms of which it is possible to parameterize the orbital motion of a test particle in the gravitational field of a central body of mass $M$ (Roy, 2003), the longitude of the ascending node $\Omega$, the argument of the pericentre $\omega$ and the mean anomaly $\mathcal{M}$ undergo secular precessions due to the even zonal harmonics $J_{\ell}, \ell=2,4,6 \ldots$ of the multipolar expansion of the Newtonian part of the gravitational potential of $M$. The general relativistic gravitomagnetic force only affects $\Omega$ and $\omega$ with the secular precessions of the Lense-Thirring effect. In principle, $\Omega, \omega$ and $\mathcal{M}$ could all be used in order to design suitable linear combinations in order to cancel out the low-degree even zonal harmonics whose classical precessions are much larger than the Lense-Thirring signals of interest. Such combinations are obtained by explicitly writing down the expressions of the residuals of $N$ orbital elements in terms of $N-1$ even zonal harmonics and the Lense-Thirring effect, considered as an entirely unmodelled feature of motion, and solving them with respect to the latter. The so obtained coefficients weighing the satellites' orbital elements depend on their semimajor axes $a$, eccentricities $e$ and inclinations $i$ : they allow to cancel out the impact of the $N-1$ even zonal harmonics considered. However, the mean anomaly cannot be used at all because it is sensitive to huge non-gravitational perturbations which especially affect the Keplerian mean motion $n=\sqrt{G M / a^{3}}$ via the indirect effects on the semi-major axis $a$. They have quadratic signature in time. Thus, only $\Omega$ and $\omega$ have been used in the so-far performed tests with the LAGEOS satellites (Ciufolini et al., 1997; 1998; Ciufolini and 
Pavlis, 2004).

\subsection{The use of the node of GP-B}

The possible use of the node of the drag-free spacecraft GP-B has been considered in (Peterson, 1997; Iorio, 2005f). The major problems come, in this case, from the fact that a nearly perfect polar orbital configuration would make the node to precess at a very low rate; for GP-B it amounts to 1136 years (Iorio, 2005f). This is a problem because one of the major timedependent tidal perturbation, i.e. the solar $K_{1}$ tide, which is not cancelled out by the linear combination approach because it is a tesseral $(m=1)$ perturbation (Iorio, 2001b), has just the same period of the satellite's node. So, over an observational time span of about one year-which is the lifetime of GP-B with the drag-free apparatus nominally working-it would resemble an aliasing superimposed secular trend (Iorio, 2002b). For example, in (Iorio, 2005f) a combination with the node of LAGEOS and GP-B has been considered: the coefficient of GP-B would amount to -398 , thus fatally enhancing such tidal bias.

\subsection{The use of the nodes of the other existing geodetic satel- lites and of CHAMP and GRACE}

Analogous conclusions can also be drawn for a possible use of CHAMP and GRACE. Indeed, let us consider, e.g., a combination with the nodes of LAGEOS, LAGEOS II, Ajisai, Starlette, Stella, CHAMP and one of the two spacecraft of the GRACE mission in order to cancel out the first six even zonal harmonics $J_{\ell}, \ell=2,4,6,8,10,12$. Apart from the coefficient of LAGEOS, which is 1 , the coefficients which weigh the nodes of the other satellites are

$$
\begin{aligned}
c_{\text {LAGEOS II }} & =0.3237, \\
c_{\text {Ajisai }} & =-0.0228, \\
c_{\text {Starlette }} & =0.0234, \\
c_{\text {Stella }} & =-0.1814, \\
c_{\text {CHAMP }} & =-12.377, \\
c_{\text {GRACE }} & =33.5348 .
\end{aligned}
$$

The slope of the gravitomagnetic trend is 3714.58 milliarcseconds per year (mas $\mathrm{yr}^{-1}$ in the following). The systematic error due to the uncancelled even zonal harmonics $J_{14}, J_{16}, J_{18}, \ldots$ amounts to $4.4 \%$, at 1-sigma level, 
according to a calculation up to degree $\ell=42$ with the variance matrix of the Earth gravity model EIGEN-CG01C (Reigber et al. 2006) which combines data from CHAMP, GRACE and ground-based measurements. Such error level is not competitive with those which can be reached by the used combination with LAGEOS and LAGEOS II and the proposed combination involving also Ajisai and Jason-1 (Iorio and Doornbos, 2005; Vespe and Rutigliano, 2005). Indeed, EIGEN-CG01C yields for them a systematic error of gravitational origin of about $6 \%$ and less than $2 \%$, respectively, at 1-sigma level. Moreover, while the error due to the geopotential is sensitive to the even zonals, at most, up to degree $\ell=20$, for such combinations, the multicombination with CHAMP and GRACE is sensitive to a much larger number of even zonal harmonics due to the inclusion of the lower altitude satellites Starlette and, especially, Stella, CHAMP and GRACE. This makes rather difficult and unreliable the evaluation of the systematic bias induced by the static part of the geopotential because the calculation of the coefficients $\dot{\Omega}_{\ell}$ of the classical secular precessions (Iorio, 2003) yield unstable results after degree $\ell \sim 40$. Thus, the $4.4 \%$ estimate of the bias due to the even zonals is probably optimistic. Another serious drawback of such multi-combination is represented by the relatively large magnitude of the coefficients (8)-(9) which weigh the nodes of CHAMP and GRACE. Indeed, they enhance the impact of all the uncancelled time-dependent perturbations among which the solar $K_{1}$ tide is one of the most powerful. The periods of the related orbital perturbations amount to -2.63 years for CHAMP and -7.23 years for GRACE. Such effects would represent serious aliasing bias over the necessarily short observational time span due to the limited lifetimes of CHAMP and GRACE with respect to the geodetic satellites.

\subsection{The use of the nodes of the other existing geodetic satel- lites}

In regard to the possibility of only using the other existing geodetic spherical satellites, mainly Ajisai, Stella and Starlette due to their long data records available, this problem has already been tackled in a number of papers, like (Casotto et al., 1990; Iorio, 2002c; 2002d). The fact that the inclusion of the other satellites in the linear combination scheme is still not competitive, although the improvements due to the first models from CHAMP and GRACE, was shown in (Iorio and Doornbos, 2005): the systematic error of gravitational origin amounts to $31 \%$ with EIGEN-CG01C. 


\subsection{The impact of the new Earth gravity models from CHAMP and GRACE on the use of a polar orbital geometry}

The impact of the most recent Earth gravity models from CHAMP and GRACE on the use of the node of a single satellite in polar orbit has been discussed in detail in (Iorio, 2005c). By using EIGEN-CG01C, it turns out that for a semimajor axis of, e.g., $8000 \mathrm{~km}$, quite larger than GP-B, CHAMP and GRACE, the systematic error due to the full spectrum of the even zonal harmonics would amount to $25 \%$ for an inclination of $88 \mathrm{deg}$. In this case the period of the $K_{1}$ tide would amount to $\sim 10^{3}$ days. Instead, for an inclination of $89.9 \mathrm{deg}$ the bias due to the even zonals would be $2 \%$ but the period of the tidal perturbation would raise to $\sim 10^{4}$ days. Also with the new terrestrial gravity field solutions the linear combination approach would fail.

\section{The proposed use of Jason-1 and Ajisai}

In a recent paper (Iorio and Doornobos, 2004), we proposed to investigate the possibility of analyzing a suitable linear combination involving the nodes of $^{3}$ LAGEOS, LAGEOS II, Ajisai and Jason-1.

\subsection{Advantages}

The advantages of the combination involving also Jason-1 and Ajisai are

- Cancellation of the first three even zonal harmonics $J_{2}, J_{4}, J_{6}$ of the geopotential along with their secular variations $\dot{J}_{2}, \dot{J}_{4}, \dot{J}_{6}$. Moreover, the systematic error due to the remaining higher degree even zonal harmonics $J_{8}, J_{10}, \ldots$ is almost model-independent: indeed, it is $\lesssim 2 \%$ (1-sigma level) according to the 2nd generation GRACE-only Earth gravity models EIGEN-GRACE02S (Reigber et al., 2005) (2\%) and GGM02S (2.7\%) and to the model EIGEN-CG01C (Reigber et al., 2006) (1.6\%). It should be mentioned that it is expected that GRACE will yield a better improvement in the knowledge of the higher degree even zonal harmonics, to which the Jason's combination is sensitive, instead of the lower degree even zonals, which mainly affect the nodenode LAGEOS-LAGEOS II combination. This means that it may happen that, in the near future, the bias due to the geopotential will reduce down to $\sim 1 \%$ for the Jason's combination in a satisfactorily

\footnotetext{
${ }^{3}$ See also (Vespe and Rutigliano, 2005) on the same topic.
} 
model-independent way, while it may remain more or less unchanged for the two-nodes LAGEOS-LAGEOS II combination. The latest results obtained with the combined model EIGEN-CG03C (Förste et al., 2005) seems to confirm this trend (Iorio, 2006d).

Finally, only the first ten even zonal harmonics (i.e. up to $J_{20}$ ) should be accounted for in the sense that the systematic error due to the geopotential does not change with the inclusion of more zonals in the calculation.

- Small coefficient, $\sim 10^{-2}$, of the node of Jason-1. This is particularly important for reducing the impact of the non-gravitational acceleration suffered by Jason-1.

- No secular or long-period perturbations of gravitational and non-gravitational origin.

\subsection{Drawbacks}

The possible weak points of this proposals are mainly the following

- The huge impact of the non-gravitational forces-mainly atmospheric drag, direct solar radiation pressure and Earth's albedo-on Jason-1 which has not a spherical shape, being endowed with solar panels. Moreover, they should be modelled in a truly dynamical way in order to avoid to absorb the Lense-Thirring effect as it would happen in the empirical reduced-dynamic approach adopted so far.

- The difficulty of getting a smooth long time series of its orbit also due to the periodical orbital maneuvers. On the other hand, it should be noted that, due to the Jason's main goal which is ocean altimetry, only the radial and transverse components of its orbit have received major attention up to now. Also the orbital maneuvers are mainly, although not entirely, in the orbital plane. Instead, the node is related to the out-of-plane component of the orbit.

However, a detailed, although preliminary, evaluation of the impact of the Jason's non-conservative forces on the entire combination has been performed in (Iorio and Doornbos, 2005). No secular effects occur. On the contrary, a relatively high-frequency (the 120-days period of the $\beta^{\prime}$ cycle related to the orientation of the orbital plane with respect to the Sun) of 
non-gravitational origin has been found. Its impact on the suggested measurement of the Lense-Thirring effect has been evaluated to be $\leq 4 \%$ over a 2-years time span (without removing such periodic signal).

Ciufolini and Pavlis (2005) do not consider the content of (Iorio and Doornbos, 2005) and do not even try to discuss it in depth. Moreover, they neither present data analysis nor numerical simulations to convincingly support their a-priori claimed infeasibility of our proposal.

\section{On the use of the mean anomaly}

In regard to the mean anomaly issue, the only work quoted by Ciufolini and Pavlis (2005) is an old, unpublished version of the preprint gr-qc/0412057 in which the mean anomaly is not mentioned at all. The same holds also for all the other subsequent versions of that preprint, some of which appeared well before the submission of (Ciufolini and Pavlis, 2005). Moreover, no other papers by us in which such an alleged explicit proposal would appear are explicitly cited in (Ciufolini and Pavlis, 2005). In fact, in the whole literature does not exist any published paper by us in which we explicitly put forth the possibility of using the mean anomaly of the LAGEOS satellites for measuring the Lense-Thirring effect. On the contrary, the use of the node and the perigee of the Earth' satellites is always explicitly mentioned in many papers where the linear approach combination is exposed and generalized to other situations (Iorio, 2002a; 2002c; 2002d; 2003; 2004; 2006a). Moreover, in (Iorio, 2005b), after a general description of the linear combination approach for the measurement of the Lense-Thirring effect, it is unambiguously written "In general, the orbital elements employed are the nodes and the perigees and the even zonal harmonics cancelled are the first N-1 low-degree ones." Ciufolini and Pavlis (2005) do not quote such sentence. In their paper it is written: "A more detailed work discussing [...] the use of the mean anomaly and other highly unfeasible proposals by Iorio (2005) will be the subjects of following paper". It is, thus, likely that the authors of (Ciufolini and Pavlis, 2005), after a careful inspection of the relevant literature, will certainly experience many difficulties in finding material for such an announced paper, at least as far as the use of the mean anomaly for measuring the Lense-Thirring effect is concerned.

Instead, it turns out that one of the authors of (Ciufolini and Pavlis, 2005) used the semimajor axis, the eccentricity and the mean anomaly of LAGEOS II in some (not explicitly released) linear combination with the nodes of LAGEOS and LAGEOS II and the perigee of LAGEOS II in some 
previous tests with the EGM96 Earth gravity model (Figure 4 and pp. 47-48 of (Ciufolini, 2001); Figure 4 and paragraph (b), pp. 2376-2377 of (Ciufolini, 2000)).

\section{The impact of the errors in the inclination on the latest test performed with the LAGEOS satel- lites}

The adopted observable is the following combination of the residuals $\delta \dot{\Omega}_{\text {obs }}$ of the rates of the nodes of LAGEOS and LAGEOS II (Iorio and Morea, 2004; Iorio, 2005a; 2006a)

$$
\delta \dot{\Omega}_{\mathrm{obs}}^{\mathrm{LAGEOS}}+c_{1} \delta \dot{\Omega}_{\mathrm{obs}}^{\mathrm{LAGEOS}} \mathrm{II} \sim \mu_{\mathrm{LT}} S_{\mathrm{LT}},
$$

where $c_{1} \sim 0.546, S_{\mathrm{LT}}=48.1$ mas $\mathrm{yr}^{-1}$ is the slope of the secular trend according to general relativity, and $\mu_{\mathrm{LT}}$ is equal to 1 in Einsteinian theory and 0 in Newtonian mechanics. The idea of using only the nodes of the LAGEOS satellites to de-couple the Lense-Thirring effect from $J_{2}$ was proposed in (Ries et al., 2003a; 2003b) in the context of the expected improvements in our knowledge of the Earth's gravity field from the dedicated GRACE mission. The linear combination approach (see also Section 2.1) was adopted for the first time by Ciufolini (1996) for his early, less precise tests with the nodes of the LAGEOS satellites and the perigee of LAGEOS II (Ciufolini et al., 1997; 1998). The combination of eq. (10) has been built up in order to cancel out the first even zonal harmonic coefficient $J_{2}$ of the multipolar expansion of the Newtonian part of the terrestrial gravitational potential, along with its time-varying part which also includes its secular variations $\dot{J}_{2}$. Up to now, the controversy about the total error budget was mainly focused on the reliable evaluation of the impact of the remaining uncancelled higher-degree even zonal harmonics $J_{4}, J_{6}, \ldots$ along with their secular

variations $\dot{J}_{4}, \dot{J}_{6}$ (Ciufolini and Pavlis 2005; Iorio 2005b; 2006b; Lucchesi 2005).

Another source of systematic error which was always explicitly neglected in the previously cited works is given by the cross-coupling between the classical precessions due to the even zonal harmonics and the uncertainties in our knowledge of the satellites' inclinations (Ciufolini et al. 1997). For the sake of clarity, let us focus on $J_{2}$ : the classical node precession induced by it is

$$
\dot{\Omega}_{J_{2}}=-\frac{3}{2}\left(\frac{R}{a}\right)^{2} \frac{n \cos i J_{2}}{\left(1-e^{2}\right)^{2}}
$$


where $n=\sqrt{G M / a^{3}}$ is the Keplerian mean motion and $R$ is the Earth's mean equatorial radius. The node orbital residuals $\delta \dot{\Omega}_{\mathrm{obs}}$ account not only for the mismodelling in $J_{2}$, whose cancellation is the goal of eq. (10), but also for the uncertainty in $i$ which yields

$$
\delta \dot{\Omega}_{i} \equiv \frac{\partial \dot{\Omega}_{J_{2}}}{\partial i} \delta i=\frac{3}{2}\left(\frac{R}{a}\right)^{2} \frac{n \sin i J_{2}}{\left(1-e^{2}\right)^{2}} \delta i .
$$

The mismodelled precessions of eq. (12) are not cancelled out by the combination of eq. (10). The total error is, thus, conservatively evaluated as

$$
\delta \mu_{i} \leq\left|\delta \dot{\Omega}_{i}^{\mathrm{LAGEOS}}\right|+\left|c_{1} \delta \dot{\Omega}_{i}^{\mathrm{LAGEOS} \mathrm{II}}\right| .
$$

An averaged root-mean-square error of 0.5 mas for both the LAGEOS satellites has been obtained by processing their data with a software like GEO-

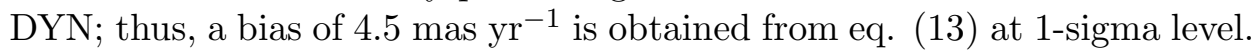
It amounts to about ${ }^{4} 9 \%$ of the Lense-Thirring effect.

This further contribution to the total error budget must be added to all the previous estimates which accounted only for the impact of $\delta \dot{J}_{\ell}$ and/or $\delta J_{\ell}, \ell=4,6, \ldots$ (Ciufolini and Pavlis 2005; Iorio 2005b; 2006b; Lucchesi 2005). In the most favorable case, i.e. a $\sim 10 \%$ gravitational bias mainly due to $\delta J_{\ell}$ only (Ciufolini and Pavlis 2005; Lucchesi 2005), the total systematic error becomes, thus, $19 \%$. If, instead, the estimates by Iorio (2006b) of the gravitational error are used, which emphasize the impact of $\delta \dot{J}_{\ell}$, the total systematic error becomes close to $30 \%$.

\section{On the combination of the nodes of LAGEOS and LAGEOS II}

In (Ciufolini and Pavlis, 2005) it is claimed that one of the authors would have put forth the idea of suitably combining the Keplerian orbital elements of the LAGEOS-type satellites in order to disentangle the Lense-Thirring effect from some of the much larger biasing secular precessions induced by the even zonal harmonics of the geopotential since the beginning of his studies on the measurability of the gravitomagnetic effect, when the LAGEOS II, launched in 1992, did not yet exist. This claim is supported by quoting from (Ciufolini, 1986; 1989): “[...] A solution would be to orbit several

\footnotetext{
${ }^{4}$ If, instead, a root-sum-square calculation is performed, $\delta \mu_{i}=$ $\sqrt{\left(\delta \dot{\Omega}_{i}^{\mathrm{LAGEOS}}\right)^{2}+\left(c_{1} \delta \dot{\Omega}_{i}^{\mathrm{LAGEOS} I \mathrm{II}}\right)^{2}}$ amounts to $6 \%$ of the Lense-Thirring effect.
} 
high-altitude, laser-ranged satellites, similar to LAGEOS, to measure $J_{2}, J_{4}$, $J_{6}$, etc., and one satellite to measure $\dot{\Omega}_{\text {Lense-Thirring." It seems more likely }}$ that this statement could, at most, mean that one should first use many satellites to accurately measure the various Earth's even zonal harmonics with a sufficiently high accuracy and, then, analyze the node only of one satellite for safely measuring its gravitomagnetic Lense-Thirring precession. However, there is no trace at all of the linear combination approach which will be introduced only in 1996 (Ciufolini, 1996) in the particular case of the nodes of LAGEOS and LAGEOS II and the perigee of LAGEOS II. Indeed, the previously quoted statement comes after a discussion of the impact of $J_{2}$ and of the higher degree even zonal harmonics on the possible use of the node only of LAGEOS; moreover, the rest of the papers (Ciufolini, 1986; 1989) deals with the supplementary configuration LAGEOS-LAGEOS X (later LAGEOS-LAGEOS III/LARES/WEBER-SAT).

The remarks about the triviality of the simple algebraic linear system of two equations in two unknowns with which the LAGEOS-LAGEOS II combination is obtained seem to be a little inappropriate because they could also be extended to the original node-node-perigee combination (Ciufolini, 1996) and the related linear algebraic system of three equations in three unknowns.

In regard to the combination of eq. (10), our papers on it are available on the Internet since April 2003. Moreover, we personally know the authors of (Ciufolini and Pavlis, 2005) having collaborated with them for some years and sent them various e-mails between April and September 2003 with our results attached; the interested reader can ask us for them. In one case (28 March 2003 and 30 March 2003), Ciufolini asked us to prepare a short table with our results in .doc format in view of a video-conference with NASA'officials to be attended in the following days by Ciufolini. A few days before, 26 March 2003, we e-mailed the .pdf of (Iorio and Morea, 2004) to Ciufolini. Later, on 7 September 2003, we discussed the impact of the GGM01C Earth gravity model on the LAGEOS-LAGEOS II combination with Pavlis.

Thus, the style and the content of the following comment present in (Ciufolini and Pavlis, 2005): "In conclusion, all the claims of Iorio (2005) are simply lacking of any rational basis: above is shown how much work was already published on this topic before Iorio (2005) even began to produce any of his paper on this topic and to rediscover some earlier results [...]. To avoid the misunderstandings of Iorio (2005), it would have just been a matter of very careful reading the previously existing literature on this subject!" are clearly inappropriate. 


\section{Conclusions}

In this paper we replied to certain criticisms by Ciufolini and Pavlis related to some issues concerning possible alternative strategies aimed to improve the quality of the currently ongoing Lense-Thirring tests with the LAGEOS satellites by using other existing active or passive spacecraft. Indeed, the combination of the nodes of LAGEOS and LAGEOS II satellites recently used represents undoubtedly a notable improvement with respect to a previous one including also the perigee of LAGEOS II. By the way, it is mainly

sensitive to $J_{4}, J_{6}$ and $\dot{J}_{4}, \dot{J}_{6}$; unless the knowledge of such part of the terrestrial gravitational field will notably be improved, the precision of the Lense-Thirring tests performed with such two-elements combination cannot be enhanced. A further source of systematic bias is represented by the uncertainties in the inclinations of the satellites. A thorough discussion about the realistically obtainable accuracy in our knowledge of such a gravitomagnetic feature is motivated not by some existing discrepancies among the general relativistic predictions for it and the so far collected and analyzed observations, but by the need of improving precise orbitography and dynamical modelling without neglecting the Lense-Thirring effect itself.

I) We demonstrated that the recent proposal by Ciufolini and Pavlis of using the orbital data from the existing terrestrial spacecraft endowed with some active mechanism of compensation of the non-gravitational perturbations like GP-B, CHAMP and GRACE is not suitable for measuring the Lense-Thirring effect both because of their too low altitude and of their nearly polar orbital geometry. Indeed, they would introduce in the resulting linear combinations much more even zonal harmonics of high degree (more than $\ell=40$ ) which would enhance the induced systematic error and make unreliable its calculation. Moreover, the polar geometry of GP-B, CHAMP and GRACE would have, as a consequence, that the coefficients with which they would enter the combinations would be larger than 1, thus enhancing various uncancelled time-dependent long-period perturbations like that due to the $K_{1}$ tide. The new Earth gravity models from CHAMP and GRACE do not alter this situation.

II) The only possibility of enhancing the reliability and the accuracy of the Lense-Thirring tests, at least with the existing spacecraft, is linked to the feasibility of the data analysis of a combination including also Jason-1 and Ajisai, although it will not be a trivial task due to the impact of the non-gravitational perturbations on Ajisai and, especially, Jason-1.

III) The claims by Ciufolini and Pavlis about an alleged proposal by us of using the mean anomaly of the laser-ranged satellites to test the Lense- 
Thirring effect have been proven to be groundless.

IV) The cross-coupling between $J_{2}$ and the errors in the satellites' inclinations lead to a further systematic bias which cannot be removed by the combination of the nodes of LAGEOS and LAGEOS II. Its impact on the measurement amounts to $9 \%$, a figure which must be added to all the previous estimates of the total error budget which only account for the even zonal harmonics and their temporal variations.

V) Finally, we have shown that the arguments by Ciufolini and Pavlis about the origin of the node-node LAGEOS-LAGEOS II combination are not correct.

\section{Acknowledgements}

I gratefully thank the anonymous referees for their efforts which greatly contributed to significantly improve this paper.

\section{References}

Ashby, N., Allison, T., 1993. Canonical planetary equations for velocitydependent forces, and the Lense-Thirring precession. Celestial Mechanics and Dynamcal Astronomy 57(4), 537-585.

Barker, B.M., O'Connell, R.F., 1974. Effect of the rotation of the central body on the orbit of a satellite Physical Review D 10(4), 1340-1342.

Casotto, S, Ciufolini, I., Vespe, F., Bianco, G., 1990. Earth satellites and Gravitomagnetic Field. Il Nuovo Cimento B 105(5), 589-599.

Ciufolini, I., 1986. Measurement of Lense-Thirring drag on high-altitude laser ranged artificial satellite. Physical Review Letters 56(4), 278-281.

Ciufolini, I., 1989. A comprehensive introduction to the LAGEOS gravitomagnetic experiment: from the importance of the gravitomagnetic field in physics to preliminary error analysis and error budget. International Journal of Modern Physics A 4(13), 3083-3145.

Ciufolini, I., 1996. On a new method to measure the gravitomagnetic field using two orbiting satellites. Il Nuovo Cimento A 109(12), 1709-1720.

Ciufolini, I., 2000. The 1995-99 measurements of the Lense-Thirring effect using laser-ranged satellites. Classical Quantum Gravity 17(12), 23692380 . 
Ciufolini, I., 2001. Gravitomagnetic phenomena due to spin, Lense-Thirring effect and its 1995-2000 measurements with Earth satellites. In: PascualSánchez, J.F., Floría, L., San Miguel, A., Vicente, F. (Eds.), Proceedings of the XXIII Spanish Relativity Meeting on Reference Frames and Gravitomagnetism. World Scientific, Singapore, pp. 35-52.

Ciufolini, I., Chieppa, F., Lucchesi, D.M., Vespe, F., 1997. Test of LenseThirring orbital shift due to spin. Classical and Quantum Gravity 14(10), 2701-2726.

Ciufolini, I., Pavlis, E.C., Chieppa, F., Fernandes-Vieira, E., PérezMercader, J., 1998. Test of General Relativity and Measurement of the Lense-Thirring Effect with Two Earth Satellites. Science 279(5359), 21002103 .

Ciufolini, I., Pavlis, E.C., 2004. A confirmation of the general relativistic prediction of the Lense-Thirring effect. Nature 431(21 October 2004), 958960. (Submitted 2 June 2004).

Ciufolini, I., Pavlis, E.C., 2005. On the measurement of the Lense-Thirring effect using the nodes of the LAGEOS satellites, in reply to "On the reliability of the so far performed tests for measuring the Lense-Thirring effect with the LAGEOS satellites" by L. Iorio. New Astronomy 10(8), 636-651.

Cugusi, L., Proverbio, E., 1978. Relativistic Effects on the Motion of Earth's Artificial Satellites. Astronomy and Astrophysics 69, 321-325.

Everitt, C.W.F., et al., 2001. Gravity Probe B: Countdown to Launch. In: Lämmerzahl C., Everitt, C.W.F., Hehl, F.W. (Eds.), Gyros, Clocks, Interferometers...: Testing Relativistic Gravity in Space. Springer, Berlin, pp. $52-82$.

Förste, C., Flechtner, F., Schmidt, R., Meyer, U., Stubenvoll, R., Barthelmes, F., König, R., Neumayer, K.H. Rothacher, M., Reigber, Ch., Biancale, R., Bruinsma, S., Lemoine, J.-M., Raimondo, J.C., 2005. A New High Resolution Global Gravity Field Model Derived From Combination of GRACE and CHAMP Mission and Altimetry/Gravimetry Surface Gravity Data. Poster g004_EGU05-A-04561.pdf presented at EGU General Assembly 2005, Vienna, Austria, 24-29, April 2005.

Iorio, L., 2001a. An alternative derivation of the Lense-Thirring drag on the orbit of a test body. Il Nuovo Cimento B 116(7), 777-789. 
Iorio, L., 2001b. Earth tides and Lense-Thirring effect. Celestial Mechanics and Dynamical Astronomy 79(3), 201-230.

Iorio, L., 2002a. Testing General Relativity With Satellite Laser Ranging: Recent Developments, invited paper at 34th COSPAR Scientific Assembly Houston, TX, 10-19 October 2002, COSPAR02-A-02121, Advances in Space Research, in press. (Preprint http://arxiv.org/abs/gr-qc/0210065).

Iorio, L., 2002b. A critical approach to the concept of a polar, low-altitude LARES satellite. Classical and Quantum Gravity 19(17), L175-L183.

Iorio, L., 2002c. Testing General Relativity with LAGEOS, LAGEOS II and Ajisai Laser-ranged Satellites. Journal of the Geodetic Society of Japan 48(1), 13-20.

Iorio, L., 2002d. Is it possible to improve the present LAGEOS-LAGEOS II Lense-Thirring experiment?. Classical and Quantum Gravity 19(21), $5473-5480$.

Iorio, L., 2003. The impact of the static part of the Earth's gravity field on some tests of General Relativity with Satellite Laser Ranging. Celestial Mechanics and Dynamical Astronomy 86(3), 277-294.

Iorio, L., 2004. Recent Developments in Testing General Relativity with Space Geodetic Techniques. In: Lynch, T.V., (Ed.), Horizons in World Physics, Vol. 245. Nova, Hauppauge, New York, pp. 1-25.

Iorio, L., 2005a. The impact of the new CHAMP and GRACE Earth gravity models on the measurement of the general relativistic Lense-Thirring effect with the LAGEOS and LAGEOS II satellites. In: Reigber, Ch., Lühr, H., Schwintzer, P., Wickert, J. (Eds.), Earth Observation with CHAMP - Results from Three Years in Orbit. Springer, Berlin, pp. 187192. (Preprint http://www.arxiv.org/abs/gr-qc/0309092).

Iorio, L., 2005b. On the reliability of the so far performed tests for measuring the Lense-Thirring effect with the LAGEOS satellites. New Astronomy 10(8), 603-615.

Iorio, L., 2005c. The impact of the new Earth gravity models on the measurement of the Lense-Thirring effect with a new satellite. New Astronomy 10(8), 616-635. 
Iorio, L., 2005d. Is it possible to measure the Lense-Thirring effect on the orbits of the planets in the gravitational field of the Sun?. Astronomy and Astrophysics 431(1), 385-389.

Iorio, L., 2005e. First preliminary evidence of the general relativistic gravitomagnetic field of the Sun and new constraints on a Yukawa-like fifth force, (Preprint http://www.arxiv.org/abs/gr-qc/0507041).

Iorio, L., 2005f. On the impossibility of using the longitude of the ascending node of GP-B for measuring the Lense-Thirring effect. General Relativity and Gravitation 37(2), 1-8.

Iorio, L., 2006a. The new Earth gravity models and the measurement of the Lense-Thirring effect. Paper presented at the Tenth Marcel Grossmann Meeting on General Relativity Rio de Janeiro, July 20-26, 2003. In: Novello, M., Perez-Bergliaffa, S. Ruffini, R.J. (Eds.), Proceedings of the Tenth Marcel Grossmann Meeting on General Relativity. World Scientific, Singapore, pp. 1011-1020. (Preprint http://www.arxiv.org/abs/gr-qc/0308022).

Iorio, L., 2006b. A critical analysis of a recent test of the Lense-Thirring effect with the LAGEOS satellites. Journal of Geodesy 80(3), 128-136. (Preprint http://www.arxiv.org/abs/gr-qc/0412057v8).

Iorio, L., 2006c. Evidence of the gravitomagnetic field of Mars. Classical and Quantum Gravity 23(17), 5451-5454.

Iorio, L., 2006d. The impact of the new Earth gravity model EIGEN-CG03C on the measurement of the Lense-Thirring effect with some existing Earth satellites. General Relativity and Gravitation 38(3), 523-527. (Preprint http://www.arxiv.org/abs/gr-qc/0505106).

Iorio, L., Morea, A., 2004. The impact of the new Earth gravity models on the measurement of the Lense-Thirring effect. General Relativity and Gravitation 36(6), 1321-1333. (Preprint http://www.arxiv.org/abs/gr-qc/0304011).

Iorio, L., Doornbos, E., 2005. How to reach a few percent level in determining the Lense-Thirring effect?. General Relativity and Gravitation 37(6), 1059-1074. (Preprint http://www.arxiv.org/abs/gr-qc/0404062).

Lense, J., Thirring, H., 1918. Über den Einfluss der Eigenrotation der Zentralkörper auf die Bewegung der Planeten und Monde nach der Einsteinschen Gravitationstheorie. Phys. Z. 19, 156-163. Translated and discussed 
by Mashhoon, B., Hehl F.W., Theiss, D.S., 1984. On the Gravitational Effects of Rotating Masses: The Thirring-Lense Papers. General Relativity and Gravitation 16(8), 711-750. Reprinted in: Ruffini, R.J., Sigismondi, C. (Eds.), 2003. Nonlinear Gravitodynamics. World Scientific, Singapore, pp. $349-388$.

Lucchesi, D.M., 2005. The impact of the even zonal harmonics secular variations on the Lense-Thirring effect measurement with the two Lageos satellites. International Journal of Modern Physics D 14(12), 1989-2023.

Mashhoon, B., Gronwald, F., Lichtenegger, H., 2001. Gravitomagnetism and the Clock Effect. In: Lämmerzahl C, Everitt, C.W.F., and Hehl, F.W. (Eds.), Gyros, Clocks, Interferometers...: Testing Relativistic Gravity in Space. Springer, Berlin, pp. 83-108.

Nordtvedt, K., 2003. Some considerations on the varieties of frame dragging. In: Ruffini, R.J., Sigismondi, C. (Eds.), Nonlinear Gravitodynamics. The Lense-Thirring Effect. World Scientific, Singapore, pp. 35-45.

Nordtevdt, K., 1988. Gravitomagnetic interaction and laser ranging to Earth satellites. Physical Review Letters 61(23), 2647-2649.

Peterson, G.E., 1997. Estimation of the Lense-Thirring precession using laser-ranged satellites. CSR-97-1, Center for Space Research, The University of Texas at Austin.

Pitjeva, E.V., 2005. Relativistic Effects and Solar Oblateness from Radar Observations of Planets and Spacecraft. Astronomy Letters 31(5), 340349 .

Reigber, Ch., Schwintzer, P., Stubenvoll, R., Schmidt, R., Flechtner, F., Meyer, U., König, R., Neumayer, H., Förste, Ch., Barthelmes, F., Zhu, S.Y., Balmino, G., Biancale, R., Lemoine, J.-M., Meixner, H., Raimondo, J.C., 2006. A High Resolution Global Gravity Field Model Combining CHAMP and GRACE Satellite Mission and Surface Gravity Data: EIGEN-CG01C. Journal of Geodesy, at press.

Reigber, Ch., Schmidt, R., Flechtner, F., König, R., Meyer, U. Neumayer, K.-H., Schwintzer, P., Zhu, S.Y., 2005. An Earth gravity field model complete to degree and order 150 from GRACE: EIGEN-GRACE02S. Journal of Geodynamics 39(1), 1-10. 
Ries, J.C., Eanes, R.J., Watkins, M.M., Tapley, B., 1989. Joint NASA/ASI Study on Measuring the Lense-Thirring Precession Using a Second LAGEOS Satellite. CSR-89-3, Center for Space Research, The University of Texas at Austin.

Ries, J.C., Eanes, R.J., Tapley, B.D., 2003a. Lense-Thirring Precession Determination from Laser Ranging to Artificial Satellites. In: Ruffini, R., Sigismondi, C. (Eds.), Nonlinear Gravitodynamics. The Lense-Thirring Effect, World Scientific, Singapore, pp. 201-211.

Ries, J.C., Eanes, R.J., Tapley, B.D., Peterson, G.E., 2003b. Prospects for an Improved Lense-Thirring Test with SLR and the GRACE Gravity Mission. In: Noomen, R., Klosko, S., Noll, C., Pearlman, M. (Eds.), Proceedings of the 13th International Laser Ranging Workshop, NASA CP 2003-212248. NASA Goddard, Greenbelt. (Preprint http://cddisa.gsfc.nasa.gov/lw13/lw_proceedings.html\#science).

Roy, A.E., 2003. Orbital Motion, Fourth edition. Institute of Physics Publishing, Bristol.

Ruggiero, M.L., Tartaglia, A., 2002. Gravitomagnetic Effects. Il Nuovo Cimento B 117(7), 743-767.

Schiff, L., 1960. On Experimental Tests of the General Theory of Relativity. American Journal of Physics 28(4), 340-343.

Sereno, M., 2003. Gravitational lensing by stars with angular momentum. Monthly Notices of the Royal Astronomical Society 344(3), 942-950.

Sereno, M., 2005a. Detecting gravitomagnetism with rotation of polarization by a gravitational lens. Monthly Notices of the Royal Astronomical Society 356(1), 381-385.

Sereno, M., 2005b. On gravitomagnetic time-delay by extended lenses. Monthly Notices of the Royal Astronomical Society 357(4), 1205-1210.

Soffel, M.H., 1989. Relativity in Astrometry, Celestial Mechanics and Geodesy. Springer, Berlin.

Stella, L. Cui, W., Chen, W., Zhang, S.N., Van Der Klis, M., Karas, V., Semerák, O., De Felice, F., Dovčiak, M., Casini, H., Montemayor, R., Morsink, S.M., Silbergleit, A.S., Wagoner, R.V., Khanna, R., Markovic, D., Lamb, F.K., 2003. Section E: Probing the Gravitomagnetic LenseThirring effect with Neutron Stars and Black Holes. In: Ruffini, R.J., 
Sigismondi, C. (Eds.), Nonlinear Gravitodynamics. The Lense-Thirring Effect. World Scientific, Singapore, pp. 235-345.

Vespe, F., Rutigliano, P., 2005. The improvement of the Earth gravity field estimation and its benefits in the atmosphere and fundamental physics, Advances in Space Research 36(3), 472-485. 